Arq. Bras. Med. Vet. Zootec., v.65, n.2, p.485-489, 2013

\title{
Comportamento ingestivo de ovinos alimentados com diferentes fontes de fibra
}

\author{
[Ingestive behavior of sheep fed with different sources of fiber] \\ M.R.P. Figueiredo ${ }^{1}$, E.O.S. Saliba ${ }^{2}$, I. Borges ${ }^{2}$, G.M.N. Rebouças ${ }^{2}$, F. Aguiar e Silva ${ }^{2}$, H.C.M. Sá ${ }^{2}$ \\ ${ }^{1}$ Pesquisadora Incaper -CRDR Centro Norte -Linhares, ES \\ ${ }^{2}$ Escola de Veterinária - Universidade Federal de Minas Gerais - Belo Horizonte, MG
}

\begin{abstract}
RESUMO
Avaliou-se o efeito de dietas distintas sobre o comportamento ingestivo (alimentação, ruminação e ócio) em ovinos. Dezesseis carneiros, castrados, confinados em gaiolas de metabolismo, com peso médio de $45,1 \mathrm{~kg}$, foram distribuídos aleatoriamente em quatro tratamentos: T1- silagem de cana + concentrado; T2- silagem de cana + concentrado $+15 \%$ de caroço de algodão; T3- silagem de cana e T4- feno de Tifton 85. Os animais foram submetidos à observação visual durante 24 horas a intervalos de 10 minutos. Houve diferença entre os tratamentos com relação aos tempos despendidos com alimentação e ócio, sendo que o tratamento com feno de Tifton 85 apresentou os mais altos valores, 6,04 h/dia, para o tempo de alimentação, e os mais baixos valores para o tempo de ócio, 7,50 h/dia.
\end{abstract}

Palavras-chave: ovinos, mastigação merícica, ócio, ruminação

\begin{abstract}
The effects of different diets on the ingestive feeding behavior in wethers (feeding, ruminating and idle) were evaluated. Sixteen wethers with an average weight of $45.1 \mathrm{~kg}$ were housed in metabolic cages and were randomly assigned to four treatments (T1- sugarcane silage + concentrate; T2- sugarcane silage + concentrate $+15 \%$ whole cottonseed; T3- sugarcane and T4- hay Tifton 85). The ingestive behavior was assessed by visual observation at 10 minute intervals for 24 hours. There were differences between treatments for time spent feeding and idle, and the treatment with Tifton 85 hay showed the highest values for feeding time, $6.04 \mathrm{~h} / \mathrm{day}$, and lower values for idle time, 7.50h/day.
\end{abstract}

Keywords: sheep, rumination chew, leisure, rumination

\section{INTRODUÇÃO}

As atividades diárias dos ovinos compreendem períodos que alternam alimentação, ruminação e ócio. Os períodos de ruminação e ócio ocorrem entre as refeições, existindo diferenças entre indivíduos quanto à duração e repetição dessas atividades, que parecem estar relacionadas às condições climáticas e de manejo, ao apetite dos animais, à exigência nutricional e, principalmente, à relação volumoso:concentrado da dieta (Silva et al., 2009).

O comportamento alimentar tem sido estudado com relação às características dos alimentos, à motilidade do pré-estômago, ao estado de vigília

Recebido em 26 de setembro de 2011

Aceito em 10 de setembro de 2012

E-mail merreg1@yahoo.com.br e ao ambiente climático. As diversidades de objetivos e condições experimentais originaram várias opções de técnicas de registro de dados, na forma de observações visuais e registros semiautomáticos e automáticos. Os parâmetros mais estudados são as descrições do comportamento ingestivo: tempo de alimentação ou ruminação, número de alimentações, períodos de ruminação e eficiência de alimentação e ruminação (Macedo et al., 2007).

Segundo Van Soest (1994), o tempo de ruminação é influenciado pela natureza da dieta e parece ser proporcional ao teor da parede celular dos volumosos. Alimentos concentrados e fenos finamente triturados ou peletizados reduzem o tempo de ruminação, enquanto volumosos com 
alto teor de parede celular tendem a elevar o tempo de ruminação. $\mathrm{O}$ aumento do consumo tende a reduzir o tempo de ruminação por grama de alimento, fator provavelmente responsável pelo aumento do tamanho das partículas fecais, quando os consumos são elevados.

O comportamento ingestivo é uma ferramenta de grande importância na avaliação das dietas, pois possibilita ajustar o manejo alimentar dos animais para obtenção de melhor desempenho produtivo. Desse modo, o objetivo deste estudo foi avaliar o comportamento alimentar em ovinos submetidos a dietas com diferentes fontes de fibra.

\section{MATERIAL E MÉTODOS}

O experimento foi realizado em laboratório de calorimetria e metabolismo animal. Foram utilizados 16 carneiros, castrados, com peso médio de $45,1 \mathrm{~kg}$, alojados em gaiolas metabólicas individuais, providas de cochos e bebedouros, recebendo água e mineralização ad libitum. Os animais foram distribuídos aleatoriamente em quatro tratamentos experimentais, sendo: $\mathrm{T} 1$ - silagem de cana + concentrado; T2- silagem de cana + concentrado $+15 \%$ de caroço de algodão; T3silagem de cana; e T4- feno de Tifton 85. A relação volumoso:concentrado das rações foi de 60:40. A composição das dietas experimentais bem como a composição bromatológica delas encontram-se dispostas na Tab. 1.

Tabela 1. Proporção dos ingredientes das dietas experimentais com base na matéria seca (MS) e composição bromatológica segundo os tratamentos $(\mathrm{T})$

\begin{tabular}{|c|c|c|c|c|}
\hline \multicolumn{5}{|c|}{ Ingrediente (\% MS) } \\
\hline Dieta $^{1}$ & $\mathrm{~T} 1$ & $\mathrm{~T} 2$ & T3 & $\mathrm{T} 4$ \\
\hline Cana-de-açúcar & 59,1 & 59,2 & 98,5 & 0,0 \\
\hline Milho & 23,0 & 19,7 & 0,0 & 0,0 \\
\hline Farelo de algodão & 16,4 & 4,6 & 0,0 & 0,0 \\
\hline Caroço de algodão & 0,0 & 15,0 & 0,0 & 0,0 \\
\hline Feno de Tifton & 0,0 & 0,0 & 0,0 & 98,5 \\
\hline Minerais e vitaminas & 1,5 & 1,5 & 1,5 & 1,5 \\
\hline Total & 100 & 100 & 100 & 100 \\
\hline \multicolumn{5}{|c|}{ Composição bromatológica } \\
\hline Matéria seca & 93,24 & 94,09 & 90,98 & 95,04 \\
\hline Matéria mineral & 6,13 & 5,94 & 6,20 & 4,41 \\
\hline Proteína bruta & 14,39 & 14,18 & 11,46 & 10,77 \\
\hline Fibra detergente neutro & 38,23 & 39,28 & 52,20 & 66,54 \\
\hline Fibra detergente ácido & 20,14 & 20,90 & 34,77 & 28,02 \\
\hline Extrato etéreo & 2,75 & 5,71 & 3,13 & 2,16 \\
\hline $\begin{array}{l}\text { Nitrogênio insolúvel em } \\
\text { detergente ácido }\end{array}$ & 1,70 & 3,48 & 3,30 & 7,59 \\
\hline $\begin{array}{l}\text { Nitrogênio insolúvel em } \\
\text { detergente neutro }\end{array}$ & 1,98 & 2,13 & 2,50 & 1,76 \\
\hline
\end{tabular}

O período experimental teve duração de 15 dias, sendo 14 dias para adaptação às dietas e à iluminação noturna e um dia para mensuração do comportamento ingestivo. A dieta foi fornecida à vontade, duas vezes ao dia, sendo ajustado o fornecimento diariamente para permitir $10 \%$ de sobras. Para mensuração do comportamento ingestivo, os animais foram submetidos à observação por quatro pessoas treinadas, em sistema de revezamento, posicionadas de maneira a não incomodar os animais. $\mathrm{O}$ comportamento ingestivo foi realizado durante o período de 24 horas, com observações de $10 \mathrm{em}$ 10 minutos das variáveis comportamentais: ócio (O), ruminação (R), ingestão (I) e mastigação (M). A média do número de mastigações merícicas por bolo ruminal e o tempo despendido de mastigação merícica por bolo ruminal foram obtidos em três períodos de duas horas, distribuídos nos horários de nove às 11 horas, 15 
às 17 horas e 19 às 21 horas, utilizando-se um cronômetro digital. A observação noturna dos animais foi realizada mediante o uso de iluminação artificial de lâmpadas incandescentes. O galpão foi mantido com iluminação artificial no período da noite, durante todo o período experimental.

Para as variáveis referentes ao comportamento ingestivo, foram utilizadas as relações propostas por Burguer et al. (2000): TMT = TAL + TRU; $\mathrm{BOL}=\mathrm{TRU} / \mathrm{MMtb} ; \mathrm{MMnd}=\mathrm{BOL} * \mathrm{MMnb}$; ERUMS = CMS/TRU; EALMS = CMS/TAL, em que: TMT (h/dia) é o tempo de mastigação total; TAL (h/dia), o tempo gasto em alimentação; TRU (h/dia), o tempo de ruminação; BOL (n\%/dia), o número de bolos ruminais; MMtb (seg/bolo), o tempo de mastigações merícicas por bolo ruminal (Polli et al., 1996); MMnb ( $\mathrm{n}^{\circ} /$ bolo), o número de mastigações merícicas por bolo; EALMS (g MS consumida/h), a eficiência de alimentação; ERUMS (g MS ruminada/h), a eficiência de ruminação; e CMS (g), o consumo diário de matéria seca.

Os dados experimentais foram analisados empregando-se o programa SAEG (Sistema..., 1997), e as médias comparadas pelo teste $\mathrm{t}$ de Student a 5\% de probabilidade.

\section{RESULTADOS E DISCUSSÃO}

A distribuição das atividades de alimentação, ruminação e ócio, em (h/dia), pode ser comparada por teste de médias na Tab. 2.

Tabela 2. Consumo de matéria seca (CMS), de fibra em detergente neutro (CFDN), tempo despendido com alimentação (TAL), ruminação (TRU), ócio (TOC) e mastigação total (TMT), eficiência de alimentação (EAL) ruminação (ERU), número de bolos ruminais (BOL), tempo gasto com mastigações por bolo ruminal (MMtb), número de mastigações por bolo ruminal (MMnb) e respectivos coeficientes de variação $(\mathrm{CV})$ de ovinos alimentados com diferentes fontes de fibra

\begin{tabular}{lccccc}
\hline Variável & T1 & T2 & T3 & T4 & CV $(\%)$ \\
\hline CMS (g/dia) & $1973,07 \mathrm{ab}$ & $2549,42 \mathrm{a}$ & $1654,40 \mathrm{~b}$ & $2252,69 \mathrm{ab}$ & 19,56 \\
CFDN (g/dia) & $1009,03 \mathrm{ab}$ & $1323,15 \mathrm{ab}$ & $878,98 \mathrm{~b}$ & $1661,35 \mathrm{a}$ & 19,26 \\
TAL (h/dia) & $4,33 \mathrm{~b}$ & $4,08 \mathrm{~b}$ & $4,46 \mathrm{~b}$ & $6,04 \mathrm{a}$ & 16,73 \\
TRU (h/dia) & 8,58 & 10,38 & 9,17 & 10,63 & 10,82 \\
TMT (h/dia) & $12,92 \mathrm{~b}$ & $14,46 \mathrm{~b}$ & $13,63 \mathrm{~b}$ & $16,67 \mathrm{a}$ & 7,86 \\
TOC (h/dia) & $11,25 \mathrm{a}$ & $9,71 \mathrm{a}$ & $10,54 \mathrm{a}$ & $7,50 \mathrm{~b}$ & 11,62 \\
EAL (gMS/h) & $459,81 \mathrm{ab}$ & $624,53 \mathrm{a}$ & $410,50 \mathrm{~b}$ & $373,20 \mathrm{~b}$ & 28,66 \\
ERUMS (gMS/h) & 234,3 & 244,41 & 181,70 & 215,70 & 22,96 \\
ERUFDN (gFDN/h) & $119,82 \mathrm{ab}$ & $126,84 \mathrm{ab}$ & $96,54 \mathrm{~b}$ & $159,06 \mathrm{a}$ & 23,44 \\
BOL (n\%/dia) & 0,18 & 0,20 & 0,19 & 0,25 & 26,60 \\
MMtb (seg/bol) & 53,02 & 54,49 & 51,8 & 44,37 & 26,83 \\
MMnb (no/dia) & $77,70 \mathrm{ab}$ & $72,68 \mathrm{ab}$ & $61,31 \mathrm{~b}$ & $88,30 \mathrm{a}$ & 22,04 \\
\hline
\end{tabular}

Médias seguidas de letras diferentes nas linhas diferem entre si pelo teste $\mathrm{t}(\mathrm{P}<0,05)$.

Tratamentos experimentais: T1 - silagem de cana + concentrado; T2- silagem de cana + concentrado + 15\% de caroço de algodão; T3- silagem de cana e T4 - feno de Tifton 85.

Foram encontradas diferenças $(\mathrm{P}>0,05)$ entre os tratamentos avaliados para os tempos despendidos em alimentação e ócio. O maior tempo de alimentação foi observado no tratamento 4, cuja fonte fibrosa foi o feno de Tifton $85 \quad(6,04 \mathrm{~h} / \mathrm{dia})$. Este tratamento apresentou o menor tempo de ócio (7,50h/dia), quando comparado aos demais tratamentos
$(\mathrm{P}<0,05)$. Tal resultado pode ser atribuído ao maior teor de fibra em detergente neutro no feno de Tifton 85 em relação à silagem de cana-deaçúcar, confirmando a influência da composição química da dieta sobre o comportamento alimentar de ovinos. 
O tempo de ruminação está altamente correlacionado ao consumo de FDN. Segundo Van Soest (1994), o aumento no teor de FDN promove aumento no tempo de ruminação devido à maior necessidade de processamento da fibra. Apesar de não apresentarem diferenças significativas entre os tratamentos para tempo despendido com ruminação, observa-se maior valor para dieta com maior teor de FDN (tratamento 4).

Schmidt et al. (2007), ao estudarem o efeito da inclusão de aditivos na ensilagem de cana-deaçúcar em bovinos de corte, encontraram valores de 3,78, 8,9, 10,98 (h/dia), respectivamente, para tempos despendidos em alimentação, ruminação e ócio, para animais alimentados com silagem de cana-de-açúcar, sem aditivos, valores esses semelhantes aos obtidos neste trabalho para a referida dieta. Da mesma forma, Macedo et al. (2007), ao avaliarem a influência do bagaço de laranja em substituição à silagem de sorgo para ovinos, encontraram valores de tempos despendidos com alimentação e ócio, de 6,04 e 7,69 h/dia, para dieta exclusiva de silagem de sorgo, valores esses semelhantes aos encontrados para a dieta com feno de Tifton. Provavelmente isso se deu pelo teor de FDN da dieta exclusiva de silagem de sorgo (70,8\%), com base na MS, semelhante ao teor de FDN da dieta com feno de Tifton (66,5\%). Gonçalves et al. (2000), ao estudarem o comportamento ingestivo de cabras leiteiras alimentadas com dietas com diferentes relações volumoso:concentrado (100:00; 80:20; 60:40 e 30:80), obtiveram, para o tratamento com $100 \%$ de volumoso, valores de 7,66 e 6,83 $\mathrm{h} /$ dia, respectivamente, para os tempos de alimentação e ruminação.

Burguer et al. (2000), ao avaliarem a influência dos níveis de concentrado nas dietas sobre o comportamento ingestivo de bezerros da raça Holandesa, observaram maior tempo despendido com ócio e menor tempo despendido com alimentação e ruminação quando se aumentou o teor de concentrado nas dietas. Esse achado se assemelha ao do presente estudo, em que o maior tempo de ócio foi observado nas dietas com concentrado (Tab. 1).

$\mathrm{O}$ aumento no fornecimento de concentrado nos animais alimentados com dietas à base de canade-açúcar como volumoso exclusivo foi suficiente para aumentar o consumo das dietas 1 e 2 se comparadas à dieta 3 . O efeito positivo do aumento do fornecimento de concentrado em dietas à base de cana-de-açúcar também foi observado por Moraes et al. (2008), que, ao avaliarem a cana-de-açúcar tratada ou não com óxido de cálcio, fornecida com diferentes níveis de concentrado $(0,0 ; 0,5 ; 1,0 \%$ do peso corporal $)$ para novilhos de corte em confinamento, relataram que o aumento do fornecimento de concentrado na dieta de cana-de-açúcar melhorou a ingestão e a digestibilidade da maioria dos nutrientes, resultando em melhor desempenho animal.

Souza et al. (2009), ao avaliarem o consumo, a digestibilidade aparente, a produção e a composição de leite e o comportamento ingestivo de vacas em lactação, em dietas contendo canade-açúcar e caroço de algodão, observaram aumento na ingestão de matéria seca, com o aumento de caroço de algodão às dietas, resultado também observado neste trabalho, para dieta com $15 \%$ de caroço de algodão. Segundo Grant (1997), o menor tamanho de partícula e a alta gravidade específica das fontes de fibra não forragem aumentam a taxa de passagem ruminal e o consumo. A fibra da cana-de-açúcar é de baixa digestibilidade, o que leva a um maior tempo de retenção no rúmen e em todo trato digestório (Preston, 1982; Rodriguez, 1995; Correa et al., 2003).

Dessa forma, mesmo que o caroço não tenha sido processado, a adição de caroço levou à substituição da FDN da cana por uma mais digestível, de maior taxa de passagem, aumentando os consumos de matéria seca e fibra em detergente neutro. Este resultado assemelhase ao de Teixeira e Borges (2005), que trabalharam com ovinos e observaram aumento no consumo de matéria seca quando incluíram caroço de algodão para animais em pastagens de Brachiaria. Warren et al. (1988) testaram níveis de $0 ; 25$ e $50 \%$ de caroço de algodão integral na dieta de ovinos e constataram que, em proporções de até $25 \%$, a ingestão e as digestibilidades da matéria seca e matéria orgânica não foram afetadas.

Avaliando-se o número de mastigações merícicas por bolo ruminal, observa-se que os maiores valores encontrados (88,30/dia) foram para o tratamento com feno de Tifton 85 , o que confirma a influência dos teores de fibra no 
comportamento ingestivo dos ovinos neste experimento.

\section{CONCLUSÕES}

Ovinos alimentados com dietas à base de feno de Tifton despenderam maior tempo com alimentação e menor tempo com ócio, quando comparados a animais com dietas à base de silagem de cana-de-açúcar.

\section{REFERÊNCIAS}

BURGUER, P.J.; PEREIRA, J.C.; QUEIROZ, A.C. et al. Comportamento ingestivo em bezerros holandeses alimentados com dietas contendo diferentes níveis de concentrado. Rev. Bras. Zootec., v.29, p.236-242, 2000.

CORREA, C.E.S.; PEREIRA, M.N.; OLIVEIRA, S.G. et al. Performance of holstein cows fed sugar cane or corn silages of different grain textures. Sci. Agric., v.60, p.621-629, 2003.

GONÇALVES, A.L.; LANA, R.P.; RODRIGUES, M.T. et al. Comportamento alimentar de cabras leiteiras submetidas a dietas com diferente relação volumoso:concentrado. In: REUNIÃO ANUAL DA SOCIEDADE BRASILEIRA DE ZOOTECNIA, 37., 2000, Viçosa, MG. Anais... Viçosa, MG. Sociedade Brasileira de Zootecnia, 2000 (CD-ROOM).

GRANT, R.T. Interactions among forages and nonforage fiber sources. J. Dairy Sci., v.80, p.1438-1446, 1997.

MACEDO, C.A.B.; MIZUBUTI, I.Y.; MOREIRA, F.B. et al. Comportamento ingestivo de ovinos recebendo dietas com diferentes níveis de bagaço de laranja em substituição à silagem de sorgo na ração. Rev. Bras. Zootec., v.36, p.1910-1016, 2007.

MORAES, K.A.K.; VALADARES FILHO, S.C.; MORAES, E.H.B.K. et al. Cana-de-açúcar tratada com óxido de cálcio fornecido com diferentes níveis de concentrado para novilhos de corte em confinamento. Rev. Bras. Zootec., v.37, p.1293-1300, 2008.

POLLI, V.A.; RESTLE, J.; SENNA, D.B. et al. Aspectos relativos à ruminação de bovinos $\mathrm{e}$ bubalinos em regime de confinamento. Rev. Bras. Zootec., v.25, p.987-993, 1996.
PRESTON, T.R. Nutritional limitation associated with the feeding of tropical forages. $J$. Anim. Sci., v.54, p.877-884, 1982.

RODRIGUEZ, N.M. Pesquisas sobre a dinâmica de fermentação ruminal e participação da digestão, realizadas no Departamento de Zootecnia da Escola de Veterinária da UFMG. In: SIMPÓSIO SOBRE EXIGÊNCIAS NUTRICIONAIS DE RUMINANTES, 1995, Viçosa, MG. Anais... Viçosa, MG: UFV, 1995, p.355-388.

SILVA, T.S.; BUSATO, K.C.; ARAGÃO, A.S.L. et al. Comportamento ingestivo de ovinos alimentados com diferentes níveis de manga em substituição ao milho. In: REUNIÃO ANUAL DA SOCIEDADE BRASILEIRA DE ZOOTECNIA, 2009, Maringá. Anais...Maringá: Sociedade Brasileira de Zootecnia, 2009. (CD ROOM).

SISTEMA de análises estatísticas e genéticas SAEG. Versão 7.1 - Viçosa: UFV, 1997. 150p.

SCHMIDT, S.P.; MARI, L.J.; NUSSIO, L.G. et al. Composição química das silagens, ingestão, digestibilidade e comportamento ingestivo. Rev. Bras. Zootec., v.36, supl., p. 1666-1675, 2007.

SOUZA, D.P.; CAMPOS, J.M.S.; VALADARES FILHO, S.C. et al. Comportamento ingestivo, consumo e digestibilidade de nutrientes, produção e composição do leite de vacas alimentadas com silagem milho ou cana-deaçúcar com caroço de algodão. Rev. Bras. Zootec., v.38, p.2053-2062, 2009.

TEIXEIRA, D.A.B.; BORGES, I. Efeito do nível de caroço integral de algodão sobre o consumo e digestibilidade aparente da fração fibrosa do feno de braquiaria (Brachiaria decumbens) em ovinos. Arq. Bras. Med. Vet. Zootec., v.57, p.229-233, 2005.

VAN SOEST, P.J. Nutritional ecology of the ruminant. 2. ed. Ithaca. NY: New York: Cornell University Press, 1994. 476p.

WARREN, H.; NEUTZE, S.A.; MORRISON, J.M. et al. The value of whole cottonseed in a wheat based maintenance ration for sheep. Austr. J. Exp. Agric., v.28, p.453-458, 1988. 